\title{
Tardive Dystonia: Clinical Spectrum and Novel Manifestations
}

\author{
R. JEFFREY DAVIS, ${ }^{1}$ JEFFREY L. CUMMINGS, ${ }^{2}$ and \\ ROBERT W. HIERHOLZER ${ }^{3}$
}

${ }_{1,2}$ Departments of Neurology and Psychiatry and Biobehavioral Sciences, UCLA School of Medicine ${ }^{2}$ Neurobehavioral Unit (691 B111) West Los Angeles. VAMC, Brentwood Division 11301 Wiltshire Boulevard L.A. CA 90073

${ }^{3}$ Psychiatry Service, Perry Point VAMC, Perry Point, New Jersey

${ }^{3}$ Albuquerque VAMC, Albuquerque, New Mexico

Correspondence to J. M. Cummings

\begin{abstract}
Tardive dystonia was identified in 25 patients: involvement of the face and neck was most common; truncal and limb dystonia were also observed. There were 3 cases of laryngospasm and 2 of spasmodic dysphonia. The latter has not been previously reported as a manifestation of tardive dystonia. In all cases, movements typical of classic tardive dyskinesia could be demonstrated. This group illustrates the variety of dystonic disorders that may occur in conjunction with tardive dyskinesia.
\end{abstract}

\section{Introduction}

Tardive dyskinesia (TD) is an iatrogenic movement disorder occurring after chronic exposure to neuroleptic agents (American Psychiatric Association, 1979). Classic TD is typically a stereotyped choreiform disorder commonly involving buccolinguomasticatory musculature and hands (Jeste and Wyatt, 1982). Other movement disorders have recently been reported as tardive manifestations including dystonia (Burke, 1982), akathisia (Weiner and Luby, 1983), myoclonus (Degurtz, 1969), and a Gilles de la Tourette-like syndrome (Klawans et al., 1978). Of these, tardive dystonia is the most common TD variant (Kang et al., 1986).

This report summarizes the clinical manifestations of tardive dystonia in 25 individuals and includes case descriptions of 3 patients with tardive laryngospasm and 2 with tardive spasmodic dysphonia, rare or previously unreported forms of tardive dystonia.

\section{Subjects}

The study was based on 25 patients referred for evaluation of a movement disorder to a Neurobehavior Clinic located in a large psychiatric hospital. A dystonic disorder was diagnosed when the patient exhibited sustained involuntary postural alterations resulting from contraction of skeletal muscle. The dystonia was identified as tardive in nature if it began after a 
minimum of 3 months of treatment with a dopamine receptor blocking agent, if alternate etiologies had been excluded, and if there was no family history of a movement disorder (Burke et al., 1982). In this report, focal dystonia refers to disorders confined to a single body region, whereas segmental dystonia is used when several body areas were affected (Fahn and Eldridge, 1976).

\section{Results}

The 25 patients included in the study comprised approximately $25 \%$ of patients with TD referred for evaluation during a 3 year period. The patients ranged in age from 25 to 70 years (mean 45.2 years); all but 2 were males, reflecting the gender composition of the hospital population. Diagnoses were established according to the Diagnostic and Statistical Manual of Mental Disorders (American Psychiatric Association, 1980) and included schizophrenia (16 cases), mood disorder ( 7 cases), and organic mental disorder ( 2 cases). Twelve (48\%) of those patients with a principal diagnosis of an idiopathic psychiatric disorder also had evidence of an organic mental syndrome. Five schizophrenic patients and 1 patient with mood disorder had histories of alcohol abuse. This diagnostic distribution is similar to that found among neuroleptic-treated patients in the hospital and among the population referred for evaluation of TD.

Nine patients had dystonic focal involvement of the face, 1 had segmental dystonia of the face and neck, and 2 exhibited facial dystonia and spasmodic dysphonia. Five patients had focal nuchal dystonia and 3 had segmental dystonic symptoms of neck and trunk or neck and adjacent limb. Two patients had focal limb dystonia and 3 had truncal dystonia. In addition to dystonic symptoms, evidence of minimal or moderate classic TD could be demonstrated in all subjects. In some cases, provocative maneuvers (e.g. distracting procedures) were necessary to demonstrate TD.

Cranial dystonia was manifested as blepharospasm (12 cases), facial grimacing (6), platysmal contraction (2), oromandibular posturing (5), sustained involuntary tongue protrusion (1), laryngospasm (3), and spasmodic dysphonia (2), some as a combination of the above. Nuchal dystonias included torticollis, $(R=1 ; L=3)$; anterocollis (3), or retrocollis (3). Limb dystonias involved primarily proximal muscular contractions resulting in shoulder elevation with abduction and torsion of the limb $(R=2, L=2)$. Truncal dystonias affected primarily the paraspinal muscles and resulted in scoliosis $(R=2, L=1)$ or lordotic exaggerations of posture (4). Generalized dystonia with dystonic posturing of the lower limbs was not observed.

Six cases representing manifestations of tardive dystonia are briefly described. Three cases with tardive laryngospasm and 2 with tardive spasmodic dysphonia represent rare or previously unreported forms of the disorder. 


\section{Case Reports}

\section{Tardive Segmental Nuchal and Limb Dystonia}

A 27-year-old right-handed man with schizoaffective disorder developed persistent left torticollis of his neck and trunk 5 years after initiation of treatment with neuroleptic agents. The dystonia was accompanied by elevation of the left shoulder and abduction and flexion of his left arm. He also complained of cramps in his hands and chest. On examination, the tone of the involved limb was slightly increased. Purposeful movements and ambulation exacerbated the dystonic posture of the neck and arm. A test dose of intramuscular diphenhydramine was without effect. Baclofen (5$20 \mathrm{mg} 4$ times per day), resperpine $(0 \cdot 1-0.25 \mathrm{mg} 3$ times per day), and diazepam (5-10 mg twice daily) provided no relief. A prosthetic neck device was eventually utilized with moderate success.

\section{Tardive Segmental Nuchal and Mixed Cranial Dystonia}

A 34-year-old right-handed man presented with major depression with mood-congruent psychotic features at age 27 . He was treated with neuroleptic agents over the next 3 years. As he improved and the neuroleptic agents were slowly discontinued, the patient noticed prolonged involuntary blinking that caused him to collide with objects and prevented him from driving. $\mathrm{He}$ also complained that his neck muscles intermittently "strained off to one side", that his tongue involuntarily protruded from his mouth, and that he frequently bit his tongue or cheek. On examination, blinking and squinting of the eyes occurred several times per minute and eye closure often persisted for two or more seconds at a time. Frequent lifting of the eyebrows also occurred. Thrusting, lifting, and lateral movements of the jaw were prominent as were tongue movements. Treatment with $2 \mu \mathrm{g}$ of benztropine meslylate 3 times daily resulted in considerable improvement.

\section{Tardive Segmental Nuchal and Cranial Dystonia with Laryngospasm}

A 54-year-old patient treated with haloperidol for a schizophrenia-like psychosis in his early 30 s presented with a 2-year history of involuntary movements of the tongue, lips, eyelids and nose. Bruxism had become constant during his waking hours. The severity of the movements had been intermittent but tolerable until 4 months prior to admission when a slowly progressive dyspnea began. On the day of hospitalization, the patient required an emergency tracheostomy to bypass severe laryngospasm. On examination, the patient was able to communicate aloud only through gutteral inflections. Choreoathetotic movements of the mouth, lips, tongue, jaw, and distal upper extremities typical of classic TD were prominent. Dystonic posturing of the neck and shoulders toward the left was also apparent. Treatment of these residual movements with clonidine $(0 \cdot 1 \mathrm{mg} / \mathrm{d})$ and reserpine $(0 \cdot 1 \mathrm{mg} 3$ times a day) was unsuccessful. 


\section{Cranial and Nuchal Dystonia with Laryngospasm}

A 40-year-old white male with a 20-year history of schizophrenia, paranoid type was admitted during acute decompensation. On examination, orobuccolingual and distal extremity dyskinesia and a pronounced right torticollis accompanied by visible hypertrophy of the left sternocleidomastoid muscle were noted. Persistent facial grimacing, hypophonia and irregular breathing without stridor or respiratory compromise were also present. Unremitting psychotic symptoms and violence required continuation of treatment with neuroleptic medications. Three months after admission, the patient abruptly developed respiratory stridor and shortness of breath. Evaluation including arterial blood gases, chest X-ray, and electrocardiogram was unremarkable. Paradoxical movement of the diaphragm was noted on fluoroscopy and indirect laryngoscopy revealed persistent laryngospasm. During periods of anxiety, all his tardive movements worsened. Trials of anticholinergic and dopaminergic depleting agents had substantial therapeutic benefit.

\section{Tardive Cranial Dystonia with Spasmodic Dysphonia}

A 60-year-old right-handed woman with bipolar affective disorder was referred for evaluation of a speech disorder. Treatment with neuroleptic agents began early in the course of recurrent depressive and hypomanic episodes. Approximately eight years previously she began to notice changes in her voice that gradually progressed to the point that talking was difficult and others found her voice odd. She noticed no other involuntary movements. She had been placed on propranolol hydrochloride $(40 \mathrm{mg}$ twice a day), but no change was noted. During neurological evaluation, attempts to speak resulted in a strained, strangled, hoarse whisper. Throughout the course of the examination, spontaneous movements of the neck in the region of the larynx and pharynx were observed. Visualization of the oropharynx revealed involuntary movements of the posterior pharyngeal structures and occasional sustained collapse of the posterior pharyngeal wall. Buccolingual dyskinesia and movements of the upper face, including blepharospasm were also present. The movements increased when the patient attempted to talk. Her breathing pattern was unremarkable and the voice abnormalities were coincident with the involuntary laryngeal and pharyngeal movements. Treatment with anticholinergic agents was recommended but the patient failed to comply.

\section{Tardive Cranial Dystonia with Spasmodic Dysphonia}

A 44-year-old woman received continuous neuroleptic treatment after developing symptoms of schizophrenia at age 28. By age 42 , prominent dyskinetic movements of the tongue and fingers were noted. Speech changes also appeared and progressed rapidly. By the time of examination, the patient exhibited strained-strangled vocalization in conjunction with dystonic jaw posturing. Volume alternated irregularly between hypo- and mega- 
phonia. Movements of classic TD were visible in the tongue, neck, and hands. A thorough evaluation revealed no alternative etiology for the spasmodic dysphonia. A trial of anticholinergic medication was without effect.

\section{Discussion}

This series of patients with tardive dystonia demonstrates the wide range of dystonic syndromes that may be observed among patients following chronic neuroleptic therapy. A predominance of involvement of the face and neck with less frequent limb and truncal symptoms was found. Two unique cases of tardive spasmodic dysphonia and 3 rare cases of tardive laryngospasm were reported.

Table 1 summarizes the principal types of dystonia manifested in patients in this series and provides comparison with previous investigations. In all studies, most of the patients had been in the fourth or fifth decades of life, younger than the average patient with $\mathrm{TD}$ but in the typical age range of patients who develop idiopathic focal dystonias. Predominance of face and neck involvement was noted in all studies whether the dystonia was focal or segmental. Abnormal neck postures, blepharospasm, and oromandibular dystonia are the most commonly reported manifestations of tardive dystonia. Generalized dystonia and focal, or segmental involvement of the lower limbs is uncommon: only 16 of 150 reported patients with tardive dystonia have had a generalized dystonic disorder and only 1 of 150 had segmental truncal and leg dystonia without involvement of other regions

TABLE 1. Summary of studies of patients with tardive dystonia

\begin{tabular}{|c|c|c|c|c|c|c|c|}
\hline Study & $\mathcal{N}$ & $\begin{array}{l}\text { Mean } \\
\text { Age }\end{array}$ & $\begin{array}{l}\text { Focal } \\
\text { Dystonia } \\
(\%)\end{array}$ & $\begin{array}{l}\text { Segmental } \\
\text { Dystonia } \\
(\%)\end{array}$ & $\begin{array}{l}\text { Generalized } \\
\text { Dystonia } \\
(\%)\end{array}$ & $\begin{array}{l}\text { Brain } \\
\text { Injury } \\
(\%)\end{array}$ & $\begin{array}{l}\text { Choreic } \\
\text { Tardive } \\
\text { Dyskinesia } \\
(\%)\end{array}$ \\
\hline $\begin{array}{l}\text { Burke et al. } \\
(1982)\end{array}$ & 42 & 34 & $9(21)$ & $27(64)$ & $6(14)$ & $6(14)$ & $10(24)$ \\
\hline $\begin{array}{l}\text { Gimenez-Rol- } \\
\text { dan et al. } \\
(1985)\end{array}$ & 9 & 36 & $2(22)$ & $6(67)$ & $1(11)$ & $3(33)$ & $4(44)$ \\
\hline $\begin{array}{l}\text { Yassa et al. } \\
\text { (1986) }\end{array}$ & 7 & 43 & $2(28)$ & $7(71)$ & - & $2(29)$ & $4(57)$ \\
\hline $\begin{array}{l}\text { Kang et al. } \\
\text { (1986) }\end{array}$ & 67 & 39 & $10(15)$ & $48(72)$ & $9(13)$ & $5(7)$ & $37(55)$ \\
\hline Current study & 25 & 45 & $19(76)$ & $6(24)$ & - & $12(48)$ & $25(100)$ \\
\hline Total (\%) & 150 & 39 & $42(28)$ & $95(63)$ & $16(11)$ & $28(19)$ & $80(53)$ \\
\hline
\end{tabular}


(Burke et al., 1982). Focal dystonia was more commonly identified in the current series while most previous studies report a predominance of segmental dystonia. Kang et al. (1986) report that many patients who present with focal dystonia later develop involvement of other areas, thus progressing from focal to segmental disorders.

Although respiratory disturbances are a well-recognized complication of TD (Jackson et al., 1980; Weiner et al., 1978; Yassa and Lal, 1986), laryngeal and pharyngeal dystonia has been rare in previously reported series. Yassa $e t$ al. (1986) reported that one of their patients had spasms of pharyngeal muscles as well as nuchal and truncal dystonia, and Yassa and Lal (1986) described a patient with severe dystonia of the neck, trunk, and limbs whose respiration was irregular and noisy with grunting and hissing. Among the patients of the current study, 3 had laryngeal dystonia, 1 sufficiently severe to require tracheostomy, and 2 had spasmodic dysphonia. The occurrence of spasmodic dysphonia in conjunction with tardive dystonic symptoms supports the evolving consensus that spasmodic dysphonia is a focal dystonia similar to other late-onset dystonic syndromes such as Meige's syndrome, torticollis, and writer's cramp (Aminoff et al., 1978; Bicknell et al., 1968; Blitzer et al., 1985).

In the present study, all patients manifested at least minimal evidence of classic choreic TD. This is in contrast to previous surveys where 24 to $57 \%$ of patients exhibited movements of classic TD in addition to tardive dystonias. The increased identification of TD in this population may reflect the routine use of provocative maneuvers to elicit the typical TD movements, or the prevalence of classic TD may vary in different patient groups. Co-existence of classic TD with dystonia aids in identifying the drug-induced nature of the movement disorder.

Demographic conclusions regarding potential risk factors for the development of tardive dystonia cannot be derived from observations of selected patients. The etiological importance of dopaminergic blocking agents is suggested by a markedly increased prevalence of dystonia among neuroleptic-treated patients compared to drug-naive populations (Friedman et al., 1987; Yassa et al., 1976). The relative youth of patients exhibiting dystonia compared with those developing classic TD indicates that younger patients may be more vulnerable to dystonic effects of neuroleptic agents. Previous investigators have found that between 7 and $33 \%$ of their patients had a history of congenital brain insult or degenerative neurologic disease (Table 1). The high frequency -48 per cent-of brain disease in the current group of patients suggests that cerebral injury should be further investigated as a possible risk factor.

Treatment of tardive dystonia is in a relatively rudimentary stage of development (Kang, 1986). Recognition of this common complication of chronic neuroleptic therapy and study of its manifold expression should facilitate the development of more successful treatment strategies. 


\section{Acknowledgements}

This project was supported by the Veterans Administration. Norene Hiekel prepared the manuscript.

\section{References}

American Psychiatric Association. (1979). Tardive dyskinesia. Task Force report 18. American Psychiatric Association, Washington, D.C.

American Psychiatric Association. (1980). Diagnostic and Statistical Manual of Mental Disorders, 3rd ed. American Psychiatric Association, Washington, D.C.

Aminoff, M. J., Dedo, H. H. and Izdebski, K. (1978). Clinical aspects of spasmodic dysphonia. Fournal of Neurology, Neurosurgery and Psychiatry, 41, 361-365.

Bicknell, J. M., Greenhouse, A. H., Pesch, R. N. (1968). Spastic dysphonia. Fournal of Neurology, Neurosurgery and Psychiatry, 31, 158-161.

Blitzer, A., Lovelace, R. E., Brin, M. F., Fahn, S. and Fink, M. E. (1985). Electromyographic findings in focal laryngeal dystonia (spastic dysphonia). (1985). Annals of Otology, Rhinology and Laryngology, 94, 591-594.

Burke, R. E., Fahn, S., Jankovic, J., Marsden, C. D., Lang, R. E., Gollomp, S. and Ilson, J. (1982). Tardive dystonia: late onset and persistent dystonia caused by antipsychotic drugs. Neurology, 32, 1335-1346.

DeVeaugh-Geiss, J. (1980). Tardive Tourette syndrome. Neurology, 30, 562-563.

Dewitz, R. (1969). Extrapyramidal motor disorders following long-term treatment with neuroleptic drugs. In "Psychotropic Drugs and Dysfunction of the Basal Ganglia", (Eds G. E. Crane and R. Gardner), Washington, D.C., U.S. Public Health Service Publication, pp 22-23.

Fahn, S. and Eldridge, R. (1976). Definition of dystonia and classification of the dystonic states. Advances in Neurology, 14, 1-5.

Friedman, J. A., Kuchavski, L. T., Wagner, R. L. (1987). Tardive dystonia in a psychiatric hospital. Journal of Neurology, Neurosurgery and Psychiatry, 50, 801-803.

Gimenez-Roldan, S., Mateo, D. and Bartolome, P. (1985). Tardive dystonia and severe tardive dyskinesia. Acta Psychiatrica Scandinavica, 71, 488494.

Jackson, I. V., Volavka, J., James, B. and Reker, D. (1980). The respiratory components of tardive dyskinesia. Biological Psychiatry, 15, 485-487.

Jeste, D. V. and Wyatt, R. J. (1982). "Understanding and Treating Tardive Dyskinesia". Guildford Press, New York.

Kang, U. J., Burke, R. E. and Fahn, S. (1986). Natural history and treatment of tardive dystonia. Movement Disorders, 1, 193-208.

Klawans, H. L., Falk, D. K., Nausieda, P. A. and Weiner, W. J. (1978). Gilles de la Tourette syndrome after long-term chlorpromazine therapy. Neurology, 28, 1064-1068.

Weiner, W. J., and Luby, E. D. (1983). Persistent akathisia following neuroleptic withdrawal. Annals of Neurology, 13, 466-467.

Weiner, W. J., Goetz, C. G., Nausieda, P. A. and Klawans, H. L. (1978). Respiratory dyskinesias: extrapyramidal dysfunction and dyspnea. Annals of Internal Medicine, 88, 327-331.

Yassa, R. and Lal, S. (1986). Respiratory irregularity and tardive dyskinesia. Acta Psychiatrica Scandinavica, 73, 506-510.

Yassa, R., Nair, V. and Dimitry, R. (1986). Prevalence of tardive dyskinesia. Acta Psychiatrica Scandinavica, 73, 629-633. 


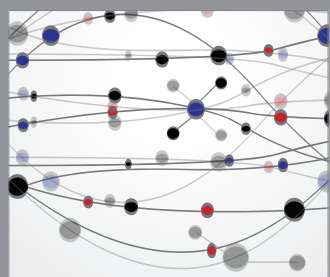

The Scientific World Journal
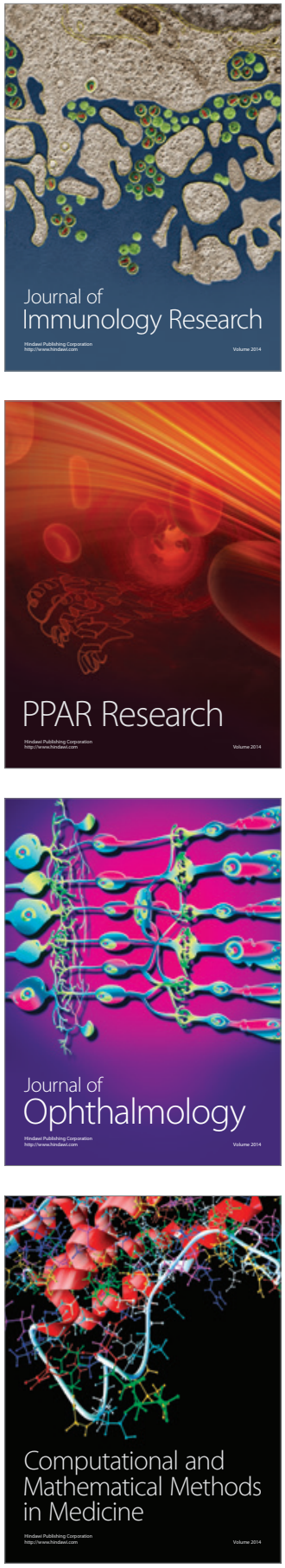

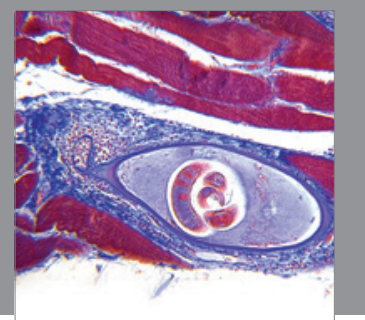

Gastroenterology

Research and Practice
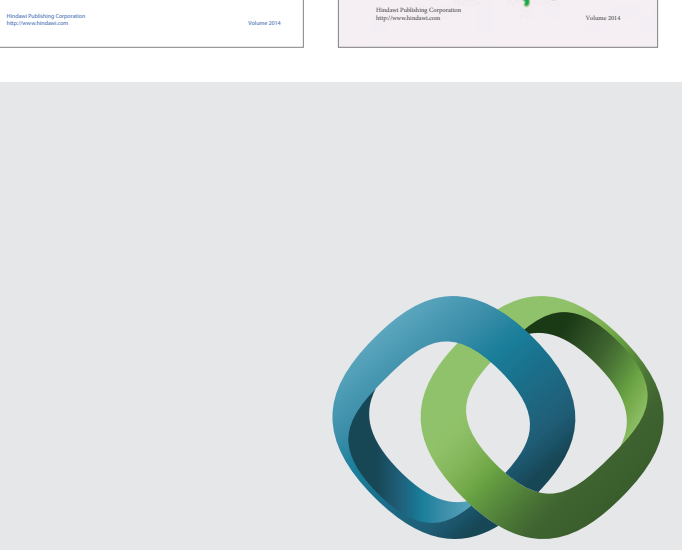

\section{Hindawi}

Submit your manuscripts at

http://www.hindawi.com
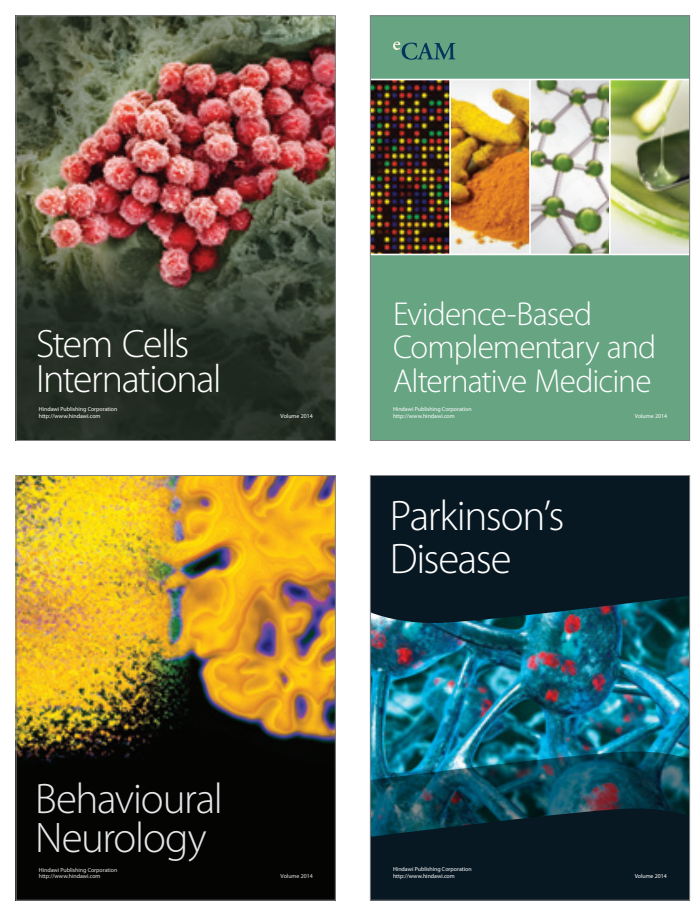

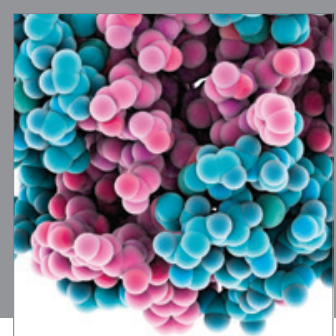

Journal of
Diabetes Research

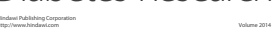

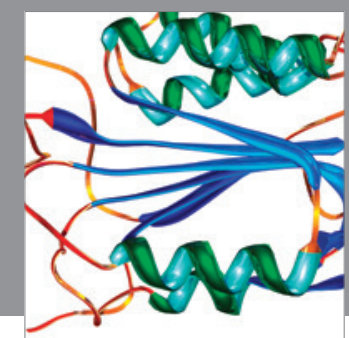

Disease Markers
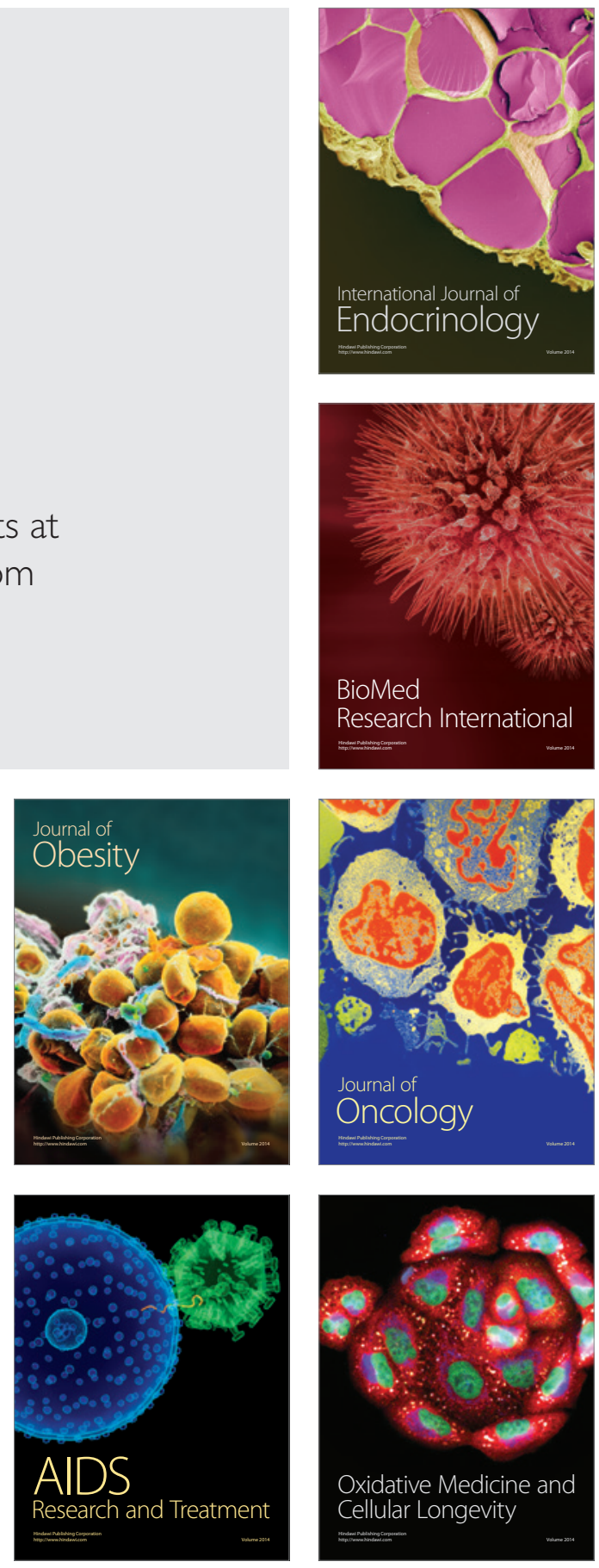